\title{
Inclusão Escolar: o Papel dos Agentes Educacionais Brasileiros
}

School Inclusion: Brazilian Educational Agents' Roles

Inclusión Escolar: el Papel de los Agentes Educativos Brasileños

Priscila Benitez

\& Camila Domeniconi

Universidade Federal de São Carlos

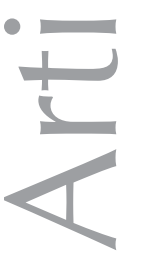

http://dx.doi.org/10.1590/1982-3703000652014

(cc) BY 
Resumo: O processo de inclusão escolar envolve diferentes agentes educacionais. A atuação conjunta entre tais agentes demonstra resultados promissores, em relação à aprendizagem social e acadêmica. O presente manuscrito teve como objetivo mapear estudos brasileiros publicados em periódicos nacionais avaliados com Qualis A1 e A2, nas áreas de Psicologia e Educação, que investigaram a atuação dos agentes educacionais em relação à inclusão escolar e discutir as principais contribuições desses achados. Foram estabelecidos quatro critérios para a realização do levantamento sistematizado. De 733 periódicos, foram selecionados 95 nacionais. Desses periódicos, 102 artigos nacionais foram recuperados com a busca por palavra-chave. 33 foram considerados resultados e classificados em estudos que contemplavam um objetivo experimental aplicado que envolvesse intervenção com qualquer agente em relação ao processo de inclusão escolar $(n=7)$ e descritivos que não tinham como objetivo a elaboração, implementação e/ou avaliação de intervenções relacionadas à inclusão escolar $(n=26)$. Oito contemplaram em alguma medida o envolvimento de distintos agentes em conjunto. São apontadas limitações e sugestões para trabalhos futuros. Os dados refletem sobre o papel dos diferentes agentes, de modo a assegurar o processo de ensino e aprendizagem dos aprendizes.

Palavras-chave: Agentes Educacionais. Inclusão Escolar. Inclusão Educacional. Práticas Inclusivas.

\begin{abstract}
School inclusion involves different educational agents. The joint work of these agents has positive results in social and academic learning. The present study aimed to depict Brazilian studies published in national journals evaluated by Qualis A1 and A2 in the Psychology and Education areas. This investigated the performance of education agents in school inclusion and discussed the main contributions of these findings. Four criteria were established for systematic review. In sum, 95 journals were selected out of a total of 733 journals. In these journals, 102 national articles were found by keyword search. A total of 33 articles were considered as results and were classified in studies that contemplated an applied experimental goal involving intervention by any agent in school inclusion $(n=7)$, and descriptive studies that did not aim at interventions for school inclusion $(n=26)$. Eight studies contemplated some involvement of these agents together. Limitations and suggestions are provided for future work. The data reflects the role of different agents in order to influence students' processes of teaching and learning.
\end{abstract}

Keywords: Educational Agent. School Inclusion. Educational Inclusion. Inclusive Practices.

Resumen: El proceso de inclusión escolar envolvió distintos agentes educativos. La actuación conjunta entre ellos mostró buenos resultados, con relación al aprendizaje social y académico. El trabajo tuvo como objetivo identificar los estudios brasileños, publicados en periódicos brasileños evaluados con Qualis A1 y A2, en las áreas de Psicología y Educación, que investigaronn la actuación de los agentes educativos con relación al proceso de inclusión escolar y discutir las principales contribuciones de los resultados. Fueran añadidos cuatro criterios para la realización de la revisión sistematizada. De 733 periódicos, fueron elegidos 95 nacionales. De estos periódicos, 102 artículos nacionales fueron buscados con las palabras-clave. Un total de 33 fueron considerados como resultados y clasificados en estudios con objetivos experimentales aplicados que tenían alguna intervención de algún agente con relación al proceso de la inclusión escolar $(n=7)$, y descriptivos que no tenían como objetivo la elaboración, implementación y/o evaluación de intervenciones relacionadas a la inclusión escolar $(n=26)$. Ocho estudios consideraron, de alguna forma, la participación de los distintos agentes en conjunto. Son presentadas las limitaciones y sugerencias para trabajos futuros. Los datos reflejan el papel de los diferentes agentes, para asegurar el proceso de la enseñanza y aprendizaje de los alumnos.

Palabras clave: Agentes Educacionales. Inclusión Escolar. Inclusión Educacional. Practicas Inclusivas. 


\section{Introdução}

Desde o início do processo de inclusão escolar, muito se tem questionado sobre como promover a inclusão escolar, de modo a assegurar a permanência ao acesso da educação de todos os estudantes envolvidos (Brasil, 2008). A publicação dos documentos normativos, como a Declaração Mundial de Educação para Todos (Unicef Brasil, 1990) e a Declaração de Salamanca (Unesco, 1994), impulsionou o desenvolvimento de práticas inclusivas no âmbito das escolas comuns e engendraram a matrícula de todos os aprendizes nesse espaço educacional.

Ainda que o processo de inclusão escolar abranja todos os alunos, de acordo com as diretrizes normativas (Brasil, 2013; Unesco, 1994; Unicef, 1990), são identificados muitos desafios para que tal processo ocorra de modo a criar práticas inclusivas efetivas em longo prazo. De modo a identificar a compreensão dos pais e dos professores sobre a inclusão escolar e social de crianças com deficiência múltipla, Silveira e Neves (2006) aplicaram uma entrevista semiestruturada, em conjunto com observações no ambiente escolar. Os resultados mostraram que tanto os pais quanto os professores acreditaram que não fosse possível ocorrer a inclusão dessas crianças no ambiente regular de ensino, devido às condições existentes na escola. Os pais, em especial, mostraram grande dificuldade para aceitar o diagnóstico dos seus filhos, expressando sentimentos de tristeza, angústia, medo e insegurança diante do diagnóstico inicial. Eles consideraram que a escola especial parece ser a melhor opção para os seus filhos, pelos seguintes motivos: as dificuldades de aprendizado apresentadas por estes; o preconceito existente no ambiente regular por parte dos outros alunos e as condições presentes na escola regular, tais como turmas com um alto número de alunos matriculados e a falta de formação dos professores para lidar com esta demanda. Os professores mostraram, em seus discursos, que a inclusão pode ser possível com alunos menos comprometidos, em relação àqueles que estavam matriculados em suas turmas (ou seja, os aprendizes que participaram deste estudo), destacaram a dificuldade de realizar qualquer atividade pedagógica com essa demanda e utilizaram os mesmos argumentos dos pais, para descreverem o ambiente regular.

Apesar de os dados de Silveira e Neves (2006) demonstrarem a realidade de uma amostra específica, em relação aos participantes, bem como ao local de coleta de dados, é possível relacionar o discurso adotado pelos pais e pelos professores no sentido de rever o espaço escolar para atender essa demanda específica de alunos. Além de que, tais achados foram similares aos relatos dos professores regulares no estudo de Mendes, Almeida e Toyoda (2011), em relação às queixas desses professores sobre a ausência de conhecimentos para trabalharem com crianças com disfunções graves, como paralisia cerebral e deficiência intelectual, no âmbito da sala regular.

Um ponto que pode ser discutido, a partir desses dados (Mendes et al., 2011; Silveira \& Neves, 2006), refere-se ao trabalho individualizado com cada aprendiz e à atuação do professor de Educação Especial inserido no contexto da sala de aula regular, para propor a adaptação dos recursos pedagógicos a serem utilizados no trabalho com cada aprendiz, a partir da colaboração com os professores regulares, como uma alternativa para enfrentar tais desafios (Mendes et al., 2011).

Portanto, para que o processo de inclusão escolar atinja todos os indivíduos e crie condições sociais e pedagógicas de ensino para todos os aprendizes, é necessário compreendê-lo enquanto processo social complexo, produto de ações estabelecidas por agentes distintos envolvidos diretamente ou indiretamente com o processo de ensino -aprendizagem, desde aqueles presentes no espaço escolar, como também presentes em seu entorno, tais como, professores (da sala comum e da educação especial), coordenadores, diretores, pais, inspetores, profissionais de serviços gerais, auxiliares de cozinha e zeladores da instituição escolar, integrantes da comunidade em geral, profissionais de saúde escolar (como: psicólogos, fonoaudiólogos, 
terapeutas ocupacionais), integrantes de instituição formadora de educadores e governo (em termos de políticas públicas) (AlmeidaVerdu, Fernandes \& Rodrigues, 2002; Benitez \& Domeniconi, 2014; Capellini \& Rodrigues, 2009; Christovam \& Cia, 2013; Gomes \& Rey, 2007; Luiz, Pfeifer, Sigolo \& Nascimento, 2012; Pelosi \& Nunes, 2009; Sant'Ana, 2005; Sousa \& Candeias, 2005).

Incluir um aprendiz na escola comum requer pensar sobre as estratégias de ensino, com a finalidade de desenvolver atividades que favoreçam tanto os aspectos sociais, como acadêmicos (ou pedagógicos). A atuação e o trabalho dos distintos agentes educacionais, com objetivos profissionais diferenciados, podem favorecer ambos os desempenhos, social e acadêmico, de todos os aprendizes, em especial, daqueles que são público-alvo da educação especial: aprendizes com deficiência (física, intelectual ou sensorial), com transtornos globais do desenvolvimento e alta habilidade/superdotação (Brasil, 2013).

A inclusão escolar, na perspectiva da educação inclusiva, demanda, portanto, a participação e o envolvimento de diferentes agentes educacionais. Quando os comportamentos desses agentes são congruentes e interdependentes no ambiente escolar, aumentam-se as possibilidades de aprendizagem, além de serem mantidos e propagados com o tempo. De tal modo, o comportamento de cada agente educacional ocorre, em função de contingências individuais. Ao somar tais contingências umas às outras, podem resultar em práticas culturais que perduram ao longo do tempo. Exemplos disso são: o comportamento do professor de Educação Especial, que realiza a adaptação da atividade para o aprendiz; o comportamento do professor de sala de aula, que ensina o aprendiz a ler; o comportamento de ler do aprendiz; o comportamento dos professores que preparam e adaptam o material didático; o comportamento dos diretores diante das políticas educacionais vigentes; o comportamento dos pais de monitorar os estudos dos filhos e tantos outros comportamentos promissores, que determinam a aprendizagem dos educandos. No entanto, alguns desses agentes nem sempre entram em contato direto uns com os outros.

Uma coletânea de estudos pode ser documentada sobre a importância da atuação dos múltiplos agentes a favor do processo inclusivo: a) o trabalho de Almeida-Verdu et al. (2002) considerou as especificidades de atuação de cada agente e assim, capacitou o coordenador pedagógico (em relação ao planejamento), os professores (no sentido de inserir discussões sobre a inclusão durante as aulas), os pais (durante as reuniões bimestrais, com orientações sobre o processo de inclusão) e os aprendizes (no que concerne à temática das diferenças); b) os achados descritos por Gena (2006) demonstrou a importância de capacitar um profissional (denominado shadow teacher) para aprimorar as interações sociais de crianças autistas incluídas em escolas comuns gregas; c) o estudo de Rolfsen e Martinez (2008) implementou e avaliou um programa psicopedagógico de orientação a pais e familiares de crianças incluídas no ensino fundamental; d) a investigação de Lima et al. (2012) criou condições para trabalhar com crianças com Síndrome de Williams, a partir do envolvimento do professor da sala de aula comum com o professor da sala experimental e das mães.

Os dados documentados nesses estudos (Almeida-Verdu et al., 2002; Gena, 2006; Lima et al., 2012; Rolfsen \& Martinez, 2008) realçaram a importância do trabalho com os agentes educacionais, para a obtenção de práticas inclusivas e ainda, referiram à atuação de novos profissionais, tais como: o shadow teacher nas escolas gregas (Gena, 2006), o teacher of the experimental class (Lima et al., 2012) e os educadores especiais, acompanhantes terapêuticos ou, ainda, tutores/facilitadores, nas escolas brasileiras (Bagaiolo, Guilhardi, \& Romano, 2005). Esses dados realçaram a importância do envolvimento de novos agentes, além do professor de sala de aula, para a aquisição de práticas inclusivas promissoras.

Dentre as diversas possibilidades de atuação dos agentes educacionais, quais intervenções têm sido implementadas por eles com os 
aprendizes da educação especial nas escolas comuns brasileiras? Com qual finalidade: social ou pedagógica? Qual estratégia de ensino empregada? Os agentes educacionais atuam em conjunto ou isoladamente? E como ocorre essa atuação? Considerando, portanto, a importância da atuação dos agentes educacionais no processo de inclusão, o presente estudo teve como objetivo mapear estudos brasileiros publicados em periódicos nacionais avaliados com Qualis A1 e A2, nas áreas de Psicologia e Educação, que investigaram a atuação dos agentes educacionais em relação à inclusão escolar e discutir as principais contribuições desses achados.

\section{Método}

\section{Fontes}

Foram pesquisados periódicos brasileiros, com conceitos A1 e A2, de acordo com a avaliação QUALIS de 2010-2012, nas áreas de Psicologia e Educação.

\section{Procedimento}

Os critérios estabelecidos para a seleção dos artigos foram: a) artigo publicado em um periódico com QUALIS A1 ou A2 nas áreas de Psicologia e Educação, b) artigo recuperado com o descritor "inclusão escolar", "inclusao escolar", "inclusão educacional"; "inclusao educacional", c) artigo publicado em um periódico que disponibilizava os textos completos online, d) abordar no texto o envolvimento em alguma medida de qualquer agente educacional com o processo de inclusão escolar.

Primeiramente, foram selecionados os periódicos qualificados com Qualis A1 e A2, nas áreas de Psicologia e Educação $(n=733)$. Na sequência, foram selecionados os periódicos nacionais, com o objetivo de atender ao objetivo proposto, em relação ao mapeamento de estudos brasileiros. Após a classificação, entre estrangeiro e nacional, foram identificados 155 periódicos nacionais.

Dos 155 periódicos, dois foram eliminados da busca, por não disponibilizarem os artigos completos em versão online (InterCom: Revista Brasileira de Ciências da Comunicação e O Percevejo - UNIRIO); 14 foram listados duas vezes, tanto na Educação, quanto na Psicologia e por essa razão foram contabilizados apenas uma vez na relação final do número de periódicos nacionais investigados, são eles: Paideia (USP), Psicologia: Reflexão e Crítica (UFRGS), Psicologia: Teoria e Pesquisa (UnB), Ágora (PPGTP/UFRJ), Educação e Sociedade, Estudos de Psicologia (Natal), Estudos de Psicologia (PUCCAMP), Estudos de Psicologia (UFRN), Psicologia em Estudo (Impresso), Psicologia em Estudo (Online), Psicologia \& Sociedade (Impresso), Psicologia \& Sociedade (Online), Revista de Psicologia: Teoria e Prática (Online), Revista Latinoamericana de Psicologia. Dos 142 periódicos, 33 foram eliminados, uma vez que alguns periódicos apresentavam dois ISSN distintos (um para a publicação online e outro para impresso), ainda que tenham sido contabilizadas duas vezes no estrato da CAPES, no presente artigo foi contabilizado como um único periódico, devido à busca na internet retornar para o mesmo periódico.

Desse contingente, 109 periódicos foram consultados com o sistema de busca por palavra-chave ("inclusão escolar", "inclusao escolar"; "inclusão educacional"; "inclusao educacional"). Como resultado, 102 artigos foram recuperados com as palavras-chave "inclusão escolar" e "inclusao escolar", dentre os quais 20 abordavam em alguma medida, a participação de algum agente educacional (pais, professores, coordenadores, dirigentes) no processo de inclusão escolar. Com as palavras-chave "inclusão educacional" e "inclusao educacional" a busca retornou 24 estudos, dentre os quais, 13 foram selecionados como resultado. As duas buscas retornaram dois estudos em comum, os quais foram considerados, uma vez como resultado. Os descritores foram selecionados de acordo com o índice de assuntos propostos pelo SCIELO, em relação a temática do processo de inclusão no âmbito escolar. A Figura 1 mostra o percurso realizado no levantamento bibliográfico sistematizado. 
Análise de dados

Os estudos que atenderam aos critérios previamente estabelecidos foram agrupados em duas categorias: 1) estudos que contemplavam um objetivo experimental aplicado que envolvesse qualquer intervenção (ou capacitação ou programa) com qualquer agente educacional em relação ao processo de inclusão escolar, 2) estudos descritivos que tinham como participantes do trabalho algum agente, porém não tinham como objetivo a elaboração, implementação e/ ou avaliação de intervenções relacionadas à inclusão escolar. Essa segunda categoria foi considerada relevante, por apresentar dados que contribuíram com sugestões para o desenvolvimento de intervenções futuras. Tais estudos utilizaram diferentes instrumentos como: entrevista, questionário e/ou observações e outros, aplicados com qualquer agente educacional.

Os estudos da categoria (1) foram descritos em termos de objetivo, método e principais resultados. Em contraste, os estudos da categoria (2) foram agrupados, fundamentalmente, em estudos que investigaram um único agente e em estudos que investigaram um número igual ou superior a dois agentes, além dos instrumentos adotados nos trabalhos.

\section{Resultados}

Os resultados foram organizados da seguinte forma: (a) características gerais dos estudos considerados como resultados nas duas categorias de análise, em termos de ano de publicação e instituições filiadas dos primeiros autores de cada artigo; (b) síntese dos sete estudos considerados como resultados na primeira categoria de análise; (c) resultados da segunda categoria de análise, em termos de número de agentes educacionais investigados em cada estudo, bem como dos instrumentos empregados.

A Figura 2 apresenta uma caracterização geral dos 33 artigos recuperados no presente estudo que atendiam as duas categorias de análise. Em termos de ano de publicação, $2012(n=7)$, 2009 e $2011(n=5)$ foram os principais anos com maior número de publicação, quando comparado aos demais anos. Ao analisar as instituições filiadas pelos pesquisadores autores de cada manuscrito, a UFSCar e a USP-SP foram as duas universidades com maior número de pesquisadores filiados $(n=5$ e $n=4$, respectivamente) e, na sequência, a PUCCAMP e Unicentro $(n=3)$. Foi utilizada a filiação apenas do primeiro autor de cada manuscrito e, caso apresentasse formação acadêmica em uma universidade diferente daquela em que atuava como docente, foi considerada na análise, a instituição atribuída em relação à atuação profissional.

Dos 33 estudos, sete abordaram alguma capacitação, intervenção e/ou programa com qualquer agente educacional, referente à temática da inclusão escolar, dentre os quais, quatro foram recuperados com a palavra-chave "inclusão escolar" e três com "inclusão educacional". O estudo de Rolfsen e Martinez (2008) propôs a implementação e avaliação de uma intervenção denominada Programa Psicopedagógico de Orientação a Pais (PPOP), destinado aos pais e familiares responsáveis por aprendizes com dificuldades

\begin{tabular}{|c|c|c|}
\hline $\begin{array}{c}\mathbf{N} \text { total de periódicos }=\mathbf{7 3 3} \\
\text { Educação } A 1-n=114 \\
\text { Educação } A 2-n=169 \\
\text { Psicologia } A 1-n=123 \\
\text { Psicologia } A 2-n=327\end{array}$ & $\begin{array}{c}\mathbf{N} \text { total de periódicos nacionais }=\mathbf{1 4 2} \\
\text { Educação } A 1-n=38 \\
\text { Educação } A 2-n=78 \\
\text { Psicologia } A 1-n=6 \\
\text { Psicologia } A 2-n=33 \\
\text { Periódicos repetidos }-n=14 \\
\text { Periódicos com dois ISSN }-\mathrm{n}=33\end{array}$ & $\begin{array}{l}\mathrm{N} \text { total de artigos nacionais } \\
\text { recuperados com busca por } \\
\text { palavra-chave } \\
\text { "inclusão escolar" }=102, \\
\text { "inclusão educacional" }=24 \mathrm{z}\end{array}$ \\
\hline & & $\begin{array}{l}\mathrm{N} \text { total de artigos nacionais } \\
\text { envolvendo agentes }=33\end{array}$ \\
\hline
\end{tabular}

Figura 1. Descrição do procedimento de coleta dos artigos. 
de aprendizagem, incluídos na primeira série do ensino fundamental de uma escola pública. Os participantes foram oito pais e seus respectivos filhos. O procedimento foi constituído por duas etapas, a destacar, (a) preparo do trabalho de campo e (b) implementação da intervenção em seis sessões e aplicação de um inventário e uma entrevista com os pais, já com os filhos foi aplicado uma avaliação de desempenho escolar. As sessões continham lições para reflexão em casa, dinâmicas e vivências. Ao longo de tais sessões, foi possível perceber mudança de comportamento dos pais, em relação a buscar maneiras de solicitar ajuda para os seus filhos. Sugestões como avaliar o nível de conhecimento dos pais antes e após a intervenção foi apontado como necessidade de aprimoramento para o programa.

Capellini et al. (2011) aplicaram um curso de formação continuada para professores sobre práticas em Educação especial e inclusiva, na área da deficiência intelectual. Participaram 20 turmas matriculadas via Ministério da Educação, com duração de 180 horas, na modalidade de educação a distância. $\mathrm{O}$ curso tinha como objetivo trabalhar assuntos teóricos sobre desenvolvimento humano, principais características da deficiência intelectual, avaliação de rendimento acadêmico, planejamento de estratégias de ensino. Tais objetivos foram trabalhados ao longo de 12 disciplinas. Os recursos adotados foram: videoconferência, material teórico, textos de apoio e atividades práticas. A equipe era composta por professores pesquisadores, 10 professores formadores e 20 tutores. Para análise dos dados da pesquisa foram consideradas três categorias: a) descrição do perfil dos cursistas, b) análise da avaliação do curso realizada por eles, c) motivos que levaram a evasão. A avaliação pelos cursistas em relação ao curso foi associada positivamente com os aspectos avaliados. Os motivos que levaram a evasão foram relacionados às limitações dos próprios cursistas. O artigo aponta para a importância do uso da educação a distância na formação de professores sobre a prática pedagógica inclusiva.

Versando, ainda, sobre cursos que visam à capacitação de professores em formação inicial e/ou continuada, em relação às práticas inclusivas, Mendes et al. (2011) discorreram sobre um programa de pesquisa, ensino e extensão, com o propósito de promover a colaboração entre os professores de educação especial com aqueles da sala de aula comum que tinham aprendizes com necessidades educativas especiais (NEE), matriculados em sala de aula. No ano de 2011, o programa encontrava-se em seu sétimo ano de execução e envolveu professores da universidade, estudantes de graduação e pós-graduação e professores do ensino comum. As aulas teóricas tinham como temática principal o estudo do ensino e consultoria colaborativo, pautadas na literatura internacional, enquanto as atividades
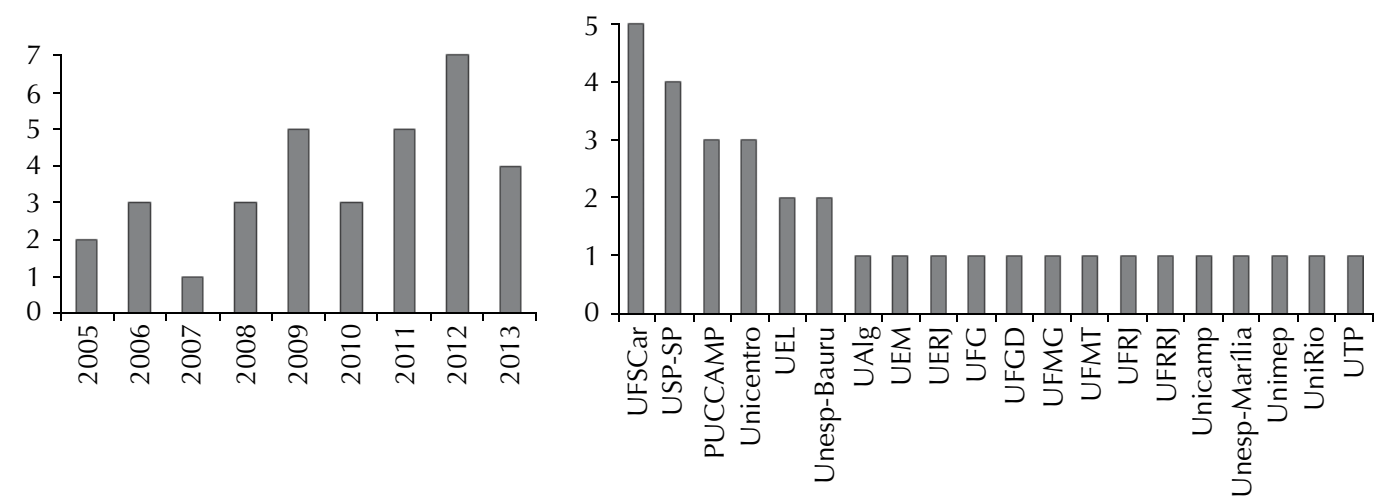

Figura 2. Caracterização geral dos 33 artigos considerados como resultados para o presente trabalho. O gráfico da esquerda apresenta o número de artigos publicados por ano e o gráfico da direita mostra a afiliação universitária do primeiro autor de cada manuscrito. 
práticas versavam na aplicação dos conhecimentos teóricos junto aos professores que tivessem aprendizes com NEE matriculados em suas respectivas salas. Inicialmente, os participantes realizavam uma entrevista com cada professor de sala, para identificar as dificuldades que encontravam para lidar com os aprendizes. Na sequência, participavam de atividades da classe comum uma vez por semana, com o objetivo de oferecer auxílio aos professores e parceria para o ensino e planejamento do conteúdo. Os resultados demonstraram a importância do emprego da perspectiva colaborativa entre educação especial e ensino comum na construção de práticas inclusivas. Ademais, os resultados do programa enfatizam os ganhos obtidos para os aprendizes em formação, como para os professores atuantes na rede.

Ainda sobre a temática da formação de professores, a rede goiana interdisciplinar de pesquisa em educação especial e inclusiva foi considerada como possível estratégia para a formação inicial e continuada dos professores para a educação inclusiva (Pereira \& Benite, 2012). Para verificar os ganhos obtidos com a rede goiana, participaram cinco professores formadores, quatro estudantes de mestrado, quatro de graduação e 12 professores da educação básica. Como instrumento, utilizaram gravações em áudio e vídeo combinado com diário de campo para analisar as interações discursivas produzidas ao longo das reuniões. Os encontros visavam à discussão de textos teóricos, versando temáticas como: conceito de inclusão e alfabetização científica. Os resultados demonstraram que as reuniões e interações, entre os distintos participantes, contribuíram para a formação de todos eles (tanto aqueles ainda em formação, como aqueles já formados).

Outro estudo (Toledo \& Vitaliano, 2012) que abordou a capacitação de professores avaliou a eficácia de um programa de formação de professores do ensino fundamental II do estado do Paraná, com o propósito de contribuir com a inclusão de aprendizes com deficiência intelectual (DI). O programa foi pautado teoricamente na premissa da inclusão educacional, no que concernem às práticas pedagógicas inclusivas. Participaram duas professoras que tinha em suas salas de aula um aprendiz com DI matriculado. $\mathrm{O}$ procedimento foi constituído por três fases. Primeiramente, ocorreu o levantamento dos conhecimentos prévios das professoras em relação à inclusão de aprendizes com DI, em conjunto com observações em sala de aula de suas práticas. Logo após, iniciaram os estudos teóricos sobre o assunto-alvo do programa com análises reflexivas das práticas adotadas em sala de aula. E, por fim, foi aplicada uma entrevista para avaliar a intervenção proposta na fase anterior. Os dados analisados apontaram para a ampliação de conhecimentos teóricos e práticos das professoras e evidenciaram a importância do ensino colaborativo entre elas e a professora da educação especial.

Tratando ainda do eixo de capacitação de professores, porém, com uma preocupação mais direcionada para o papel do psicólogo escolar na consultoria colaborativa com os professores, Silva e Mendes (2012) avaliaram um programa de intervenção preventiva, elaborado com base na consultoria colaborativa escolar e de suporte comportamental positivo, no sentido de prevenir e reduzir problemas de comportamentos. Participaram três professoras de sala de aula do ensino fundamental e todos os aprendizes da sala. $\mathrm{O}$ procedimento foi composto por: a) pré-teste (medidas antes da intervenção), b) aplicação do programa com os professores e os aprendizes, c) pós-teste. O programa de intervenção foi composto por 19 reuniões semanais com as professoras, em módulos teóricos (a destacar, manejo de conteúdo, alterações no ambiente físico e outros). Entre as reuniões, foram propostas visitas nas salas de aulas, para que o psicólogo pudesse contribuir na realização das intervenções planejadas durante as reuniões. Com os aprendizes, foi realizado um treinamento de habilidades sociais, como estratégia de prevenção dos problemas de comportamento. Os dados demonstraram, por um lado, redução nos problemas de comportamento dos aprendizes e, por outro, uma perspectiva de trabalho do psicólogo escolar direcionado na prevenção de problemas de comportamento na escola. 
Bardy, Hayashi, Schlünzen e Seabra Júnior (2013) propuseram analisar o uso de objetos de aprendizagem (OA) para a formação de professores em contextos inclusivos, na modalidade a distância. Participaram professores de um curso sobre tecnologia assistiva oferecido pelo Ministério da Educação. Os achados foram descritos em duas categorias de análise, uma relacionada ao módulo que visava o ensino sobre $\mathrm{OA}$ e a outra, com base nos relatórios finais entregues a coordenação do curso. Os OAs podem beneficiar a aprendizagem dos conteúdos disciplinares, além de servirem como estratégia para a inclusão de pessoas com deficiência e permitirem o trabalho com conteúdos acadêmicos diversificados. A modalidade a distância foi avaliada positivamente pelos professores, configurando uma possibilidade concreta de formação continuada, a partir das trocas de experiências entre os professores cursistas, além de propiciar a relação entre os conteúdos teóricos aos práticos e inovação na criação e desenvolvimento de estratégias pedagógicas.
Apesar dos sete estudos (Bardy et al., 2013; Capellini et al., 2011; Mendes et al., 2011; Pereira \& Benite, 2012; Rolfsen \& Martinez, 2008; Silva \& Mendes, 2012; Toledo \& Vitaliano, 2012) abordarem, em alguma medida, a capacitação e formação de algum agente educacional (como: pais e professores) em relação à temática da inclusão escolar, nenhum deles objetivou o trabalho em conjunto entre dois ou mais agentes, vislumbrando o ensino de conteúdos acadêmicos para aprendizes da educação especial matriculados na escola comum.

Em relação aos resultados analisados na categoria (2) que não tinham a finalidade de elaborar, implementar e/ou avaliar intervenções envolvendo qualquer agente, no que concerne à inclusão escolar, os dados mostraram que os trabalhos desenvolvidos com um único agente, em sua grande parte, listaram contribuições particulares de acordo com as necessidades de cada agente. Especificamente, em relação às demandas apresentadas pelos professores, foram atribuídas dificuldades de socialização dos aprendizes

Tabela. Descrição dos resultados analisados na categoria (2).

\begin{tabular}{|c|c|c|c|}
\hline \multicolumn{2}{|l|}{ Agentes educacionais isolados } & \multicolumn{2}{|c|}{ Agentes educacionais em conjunto } \\
\hline Estudos & Agentes & Estudos & Agentes \\
\hline Silva e Leme (2009) & Diretor & Sant'Ana (2005) & Professores e diretores \\
\hline Musis e Carvalho (2010) & $\begin{array}{l}\text { Graduandos em } \\
\text { Pedagogia }\end{array}$ & $\begin{array}{c}\text { Sousa e Candeias } \\
\qquad(2005)\end{array}$ & $\begin{array}{l}\text { Professores e profissionais da } \\
\text { equipe de saúde escolar }\end{array}$ \\
\hline Luiz et al. (2012) & Famílias & Lacerda (2006) & $\begin{array}{l}\text { Professores e intérpretes de } \\
\text { língua de sinais }\end{array}$ \\
\hline \multirow{5}{*}{$\begin{array}{l}\text { Aspilicueta, Leite, Rosa e Cruz (2013); } \\
\text { Capellini e Rodrigues (2009); Cruz, } \\
\text { Schneckenberg, Tassa e Chaves (2011); } \\
\text { Farias, Maranhão e Cunha (2008); } \\
\text { França e Munford (2012); Gomes e } \\
\text { Mendes (2010); Gomes e Souza (2012); } \\
\text { Guarinello, Berberian, Santana, Massi e } \\
\text { Paula, (2006); Leonardo, Bray e Rossato } \\
\text { (2009); Monteiro e Manzini (2008); Naiff, } \\
\text { Ferreira e Naiff (2013); Pelosini e Nunes } \\
\text { (2009); Rodrigues, Moreira e Lerner } \\
\text { (2012); Sampaio (2006); Vitaliano (2007) }\end{array}$} & Professores & Gomes e Rey (2007) & $\begin{array}{l}\text { Diretora, vice-diretora, } \\
\text { coordenadora pedagógica, } \\
\text { inspetoras, professores }\end{array}$ \\
\hline & & $\begin{array}{l}\text { Cruz, Aspilicueta e } \\
\text { Leite (2009) }\end{array}$ & $\begin{array}{l}\text { Diretora, coordenadora } \\
\text { pedagógica, funcionárias de } \\
\text { serviços gerais, professores }\end{array}$ \\
\hline & & $\begin{array}{l}\text { Sekkel, Zanelatto e } \\
\text { Brandão (2010) }\end{array}$ & $\begin{array}{l}\text { Professores, coordenadora e } \\
\text { profissionais de apoio }\end{array}$ \\
\hline & & $\begin{array}{c}\text { Oliveira, Antunes, } \\
\text { Rocha e Teixeira } \\
\text { (2011) }\end{array}$ & $\begin{array}{l}\text { Graduandos de Biologia, } \\
\text { Química e Física }\end{array}$ \\
\hline & & $\begin{array}{c}\text { Christovam e Cia } \\
\text { (2013) }\end{array}$ & Pais e professores \\
\hline
\end{tabular}


incluídos e preocupações com o conteúdo disciplinar, no que concernem às possíveis adaptações curriculares (França \& Munford, 2012; Gomes, \& Mendes, 2010; Rodrigues, Moreira \& Lerner, 2012). Em contrapartida, os estudos que investigaram, em alguma medida, dois ou mais agentes demonstraram preocupações que perpassaram as necessidades específicas e individuais de cada um deles, como em relação aos aspectos de infraestrutura, formação continuada, adequação de recursos pedagógicos, apoio da família (Christovam \& Cia, 2013; Leonardo, Bray \& Rossato, 2009; Sant'Ana, 2005), colaboração e articulação entre os professores e os profissionais de saúde (Sousa \& Candeias, 2005) e posicionamento de cada agente educacional (Gomes \& Rey, 2007). A Tabela mostra a sistematização proposta para a categoria (2). De 33 estudos considerados como resultados, 26 enquadraram nessa categoria de análise.

Dentre os principais agentes educacionais envolvidos com o processo de inclusão escolar, de acordo com o mapeamento realizado nesse trabalho, destacam-se: professores $(n=28)$, diretores $(n=4)$, coordenadores pedagógicos $(n=3)$, pais $(n=3)$, professores em formação inicial - graduandos $(n=2)$, funcionários responsáveis por serviços gerais $(n=1)$, inspetores $(n=1)$, interpretes de línguas de sinais $(n=1)$, profissionais da equipe de saúde escolar $(n=1)$, profissionais de apoio $(n=1)$ e vice-diretores $(n=1)$. A maioria dos estudos investigou mais de um agente educacional, por exemplo, Sant'Ana (2005) entrevistou 10 professores e seis diretores, por essa razão, para a realização do cálculo apresentado foi atribuído um ponto para cada agente (um ponto para professores e um ponto para diretores), apesar de ser um mesmo estudo.

Os instrumentos de pesquisa utilizados nos estudos analisados na categoria (2), foram listados: questionários $(n=8)$, entrevistas sem especificações $(n=6)$, entrevistas semiestruturadas $(n=4)$, observações $(n=4)$, diário de pesquisa $(n=3)$, escalas $(n=2)$, registro em áudio e vídeo $(\mathrm{n}=2)$, sistemas conversacionais $(n=2)$, questionário semiestruturado $(n=2)$, análise documental $(n=1)$, entrevistas coletivas $(n=1)$, entrevista de aprofundamento $(n=1)$, observação participante $(n=1)$, relatos de professores $(n=1)$, tarefas de evocação livre $(n=1)$. O mesmo critério para o cálculo dos agentes foi adotado para os instrumentos. Não foram inseridos sete estudos da categoria (1). E o recurso diário de pesquisa englobou o recurso notas de campo.

Esses dados demonstram a preocupação das pesquisas nacionais para auxiliar os professores, enquanto agente educacional envolvido com a inclusão escolar, além do uso exaustivo, em grande parte, de questionários e entrevistas para realizar tal auxílio.

\section{Discussão}

O presente estudo teve como objetivo mapear estudos brasileiros publicados em periódicos nacionais avaliados com Qualis A1 e A2, nas áreas de Psicologia e Educação com a temática sobre a atuação dos agentes educacionais em relação ao processo de inclusão escolar e discutir as principais contribuições desses achados, no sentindo de iniciar reflexões, ainda que principiantes sobre o envolvimento e a atuação dos diferentes agentes educacionais considerando as diversas possibilidades de atuação dos agentes educacionais, com destaque para o ensino de comportamentos sociais e acadêmicos.

Os resultados classificados nas duas categorias propiciaram discutir os dados analisados no levantamento proposto, em relação às principais contribuições das produções nacionais, no que diz respeito ao processo de inclusão escolar, questionamentos que ainda carecem de investigações mais acuradas para serem desenvolvidas, a fim de planejar e implementar práticas inclusivas promissoras que possam gerar contribuições para o desenvolvimento de estudos posteriores.

Os dados da categoria (1) replicaram os achados da segunda categoria, em relação aos estudos preocupados com a formação do professor (Bardy et al., 2013; Capellini et al., 2011; Mendes et al., 2011; Pereira \& 
Benite, 2012; Silva \& Mendes, 2012; Toledo $\&$ Vitaliano, 2012), a favor do planejamento de práticas inclusivas. Isso sugere repensar no papel do professor nesse contexto e refletir sobre suas atuais demandas de trabalho, em busca de formações continuadas que favoreçam atuações pedagógicas promissoras para ensinar todos os aprendizes.

Discutir sobre a formação de professores requer pensar desde a formação inicial, no âmbito das universidades, até as condições de trabalho dos professores, tal como o desenvolvimento profissional (Jesus, Barreto \& Gonçalves, 2011). Contudo, grande parte dos cursos de formação inicial do professor (ou seja, as licenciaturas), especialmente, nas áreas de Biologia, Química e Física, ainda carecem de conteúdos que discutam estratégias inclusivas pedagógicas que assegurem o desempenho acadêmico dos aprendizes com NEE (França \& Munford, 2012; Oliveira et al., 2011).

Os resultados analisados em ambas as categorias, apontaram para a necessidade de repensar o papel do professor em sala de aula, no sentido de rever a estrutura do seu trabalho, de modo a criar condições para que sejam responsáveis pelas tomadas de decisão no âmbito escolar, em conjunto com os demais agentes, bem como decidirem conteúdos destinados para as suas formações continuadas (Capellini \& Rodrigues, 2009; Cruz et al., 2011; Gomes \& Rey, 2007; Gomes \& Souza, 2012; Leonardo et al., 2009; Luiz et al., 2012; Musis \& Carvalho, 2010; Sant'Ana, 2005; Vitaliano, 2007).

Um meio de compreender a formação continuada de professores, de acordo com os trabalhos revisados no Grupo de TrabaIho 15 - Educação Especial da Associação Nacional de Pós-Graduação e Pesquisa em Educação (ANPED), refere-se à formação interativa individualizada, com modalidades presenciais e a distância, tal como grupos de estudo (Jesus et al., 2011). Esse modelo de educação continuada com os professores dialoga com os achados de Pereira e Benite (2012), ao descreverem sobre a atuação da rede goiana interdisciplinar de pesquisa em educação especial e inclusiva na modalidade presencial, assim como com os dados de Mendes et al. (2011) e Toledo e Vitaliano (2012), que apresentaram um programa de formação na perspectiva do ensino colaborativo de modo presencial, e Rolfsen e Martinez (2008) e Bardy et al. (2013) na modalidade a distância. Independentemente do recurso utilizado (presencial, individualizado, grupo de estudo, a distância) para a realização da formação continuada, um comum consenso foi encontrado entre tais estudos, no que diz respeito à importância de capacitações continuadas em prol de práticas inclusivas promissoras no âmbito da escola comum. Todavia, questões sobre os procedimentos de avaliação dessas capacitações ainda carecem de maior clareza, especialmente, em relação à efetividade de cada uma delas em termos práticos, em longo prazo. Como, por exemplo, estudos que adotem estratégias de follow-up para garantir uma medida de manutenção da aprendizagem, após a realização de cada capacitação.

Nesse escopo de discussão, outra forma plausível de capacitar os agentes educacionais, com o propósito de desenvolver práticas inclusivas, poderia ser por intermédio de uma programação de ensino detalhada em pequenas unidades, com aumento gradual da dificuldade de conteúdo a ser ensinada para cada agente, de acordo com o ritmo de aprendizagem individual de cada um deles (Zanotto, 2000), além de atividades que propiciem articulações diretas com as respectivas rotinas, em seus ambientes de atuação.

Considerando o papel da escola como aquela que contribui no processo de desenvolvimento dos aprendizes (Rodrigues et al., 2012), a inclusão escolar ainda apresenta vários desafios para a sua efetiva implementação na escola comum. Desafios como infraestrutura adequada para a implementação de projetos inclusivos, especialmente, a necessidade de material para a efetiva elaboração de atividades pedagógicas diferenciadas, direcionamento adequado para o trabalho e um número menor de aprendizes em sala de aula e a ampliação no quadro da equipe escolar, com destaque para o professor de educação 
especial (Capellini \& Rodrigues, 2009; França \& Munford, 2012; Gomes, \& Souza, 2012; Leonardo et al., 2009; Rodrigues et al., 2012; Sant'Ana, 2005; Silva \& Leme, 2009).

Como alternativa remediativa para tais desafios, as estratégias de ensino propostas envolvem formação de uma equipe multidisciplinar, formação continuada, infraestrutura e recursos pedagógicos adequados, realização do Plano de Ensino Individualizado (PEI) e auxílio da família durante esse processo. $\mathrm{O}$ auxílio da família adquire relevante importância, visto que a escola inclusiva requer a efetivação de currículo adequado, adaptado ou modificado, quando necessário, e uma prática pedagógica flexível, com arranjos e adaptações que favoreçam tanto o aproveitamento, quanto o ajuste sócio-educacional do aprendiz com NEE (Sanches \& Oliveira, 2011; Sant'Ana, 2005). Essas estratégias de ensino podem ser descritas como uma classe de comportamentos esperados pelos agentes, em relação a cada aprendiz incluído.

De modo que esses desafios possam ser suprimidos a partir do estabelecimento de políticas públicas, da equipe escolar em sua totalidade e do envolvimento dos pais (Capellini \& Rodrigues, 2009; Christovam \& Cia, 2013; Sant'Ana, 2005), torna-se fundamental identificar e compreender o papel de cada agente educacional envolvido no espaço escolar, como proposto no estudo de Silva e Mendes (2012) ao sugerir a atuação do psicólogo escolar na consultoria colaborativa com o professor de sala de aula; assim como o papel dos pais em relação à monitoria dos estudos dos seus filhos, a organização de rotinas, de estratégias promissoras para a aquisição do comportamento de estudar de seus filhos, a fim de propiciar interações positivas, por intermédio de práticas parentais positivas (Christovam \& Cia, 2013); o papel do professor de educação especial no que diz respeito aos serviços pedagógicos e orientações quanto ao uso de recursos no processo de ensino e aprendizagem (Brasil, 2008); o papel do professor de sala de aula, em relação ao trabalho com os conteúdos estabelecidos pelos Parâmetros Curriculares Nacionais-PCNs (Brasil, 1997) e tantos outros papéis de diferentes agentes educacionais envolvidos, para garantir que o processo de inclusão escolar ocorra de modo a produzir resultados promissores em relação ao desempenho acadêmico e social do aprendiz incluído.

Além dos agentes presentes na rotina escolar, é imprescindível ressaltar o papel dos novos profissionais (para exemplificar - shadow teacher, teacher of the experimental class, educadores especiais, acompanhantes terapêuticos, tutores/facilitadores) necessários para que a inclusão escolar produza dados concretos referentes aos desempenhos acadêmicos e sociais dos alunos incluídos nesse processo (Bagaiolo et al., 2005; Gena, 2006; Lima et al., 2012). Deste modo, são propostas sugestões de operacionalização das distintas atuações previstas para cada agente, tal qual de repensar na equipe escolar, sobretudo, na inserção destes novos profissionais, de modo a garantir a articulação e a colaboração entre eles, a partir de capacitações que visem, principalmente, à elaboração de estratégias inclusivas em conjunto, de modo compartilhado na proposição de intervenções educativas pedagógicas (Gomes \& Rey, 2007; Lacerda, 2006; Luiz et al., 2012; Pelosi \& Nunes, 2009; Sant'Ana, 2005; Sousa \& Candeias, 2005).

O levantamento bibliográfico sistematizado evidenciou que, apesar da necessidade da atuação direcionada para o favorecimento da inclusão tanto do ponto de vista social quanto acadêmico, a maioria das intervenções conduzidas pelos agentes educacionais, de acordo com a literatura, prevê o ensino de comportamentos sociais, corroborando as premissas de estudos prévios (Gena, 2006; Lima et al., 2012; Sanches \& Oliveira, 2011; Silva \& Mendes, 2012). Dos sete estudos da categoria (1), três (Bardy et al., 2013; Capellini et al., 2011; Pereira \& Benite, 2012) capacitaram, em termos teóricos, os professores, em relação às práticas inclusivas, em sua generalidade e quatro deles (Mendes et al., 2011, Rolfsen \& Martinez, 2008; Silva \& Mendes, 2012; Toledo \& Vitaliano, 2012) criaram condições para capacitarem os agentes (professores e pais) na interação com o aprendiz com NEE. Dentre estes quatro estudos, a finalidade da capacitação não estava direcionada ao ensino de um comportamento acadêmico específico. 
No que tange aos aspectos acadêmicos, sobretudo, habilidades acadêmicas básicas como leitura e escrita, que servem como pré-requisitos para o acesso a outras disciplinas escolares, espera-se que os agentes atuem na programação e na aplicação de intervenções que garantam a aquisição destas habilidades pelos aprendizes incluídos na escola comum. Ensinar habilidades básicas de leitura e escrita para qualquer aprendiz está previsto como objetivo terminal dos anos iniciais do ensino fundamental, de acordo com os PCNs (Brasil, 1997). Apesar da regulamentação e da importância dessa atuação direcionada à aprendizagem acadêmica do aprendiz especial, a literatura mostra ainda carência na investigação com este foco.

Refletir sobre condições que garantam o acesso e a permanência do aprendiz com NEE na escola comum requer desenvolver estratégias tanto no âmbito social, quanto acadêmico, de modo a alcançar os objetivos propostos pela educação escolar brasileira, combase na Declaração Mundial de Educação para Todos (Unicef, 1990). Dada à carência de estudos que garantam a aprendizagem acadêmica dos aprendizes com NEE incluídos na rede, particularmente aqueles que apresentam qualquer comprometimento de origem cognitiva, como a deficiência intelectual e os transtornos globais do desenvolvimento, em especial, o transtorno do espectro autista, ou mesmo, no caso da deficiência múltipla, por meio dos agentes educacionais envolvidos no processo, sugere-se que estudos futuros desenvolvam intervenções ou capacitações que garantam, ainda que de modo preliminar, o ensino de habilidades acadêmicas básicas para essa demanda.

O processo de aprendizagem, especialmente quando se trata de práticas inclusivas favorecedoras do comportamento de aprender, pode ser compreendido a partir do envolvimento dos distintos agentes educacionais (Almeida-Verdu et al., 2002; Benitez \& Domeniconi, 2014). Garantir a congruência e o trabalho cooperativo entre tais agentes parece ser uma condição importante para o desenvolvimento de práticas inclusivas (Gusmão, Martins \& Luna, 2011). Mesmo que seja importante garantir a capacitação e o envolvimento direto desses agentes, os dados mostraram baixo número de pesquisas $(\mathrm{n}=8)$ que investigou dois ou mais agentes em um mesmo estudo. Ainda que esses oito estudos tenham proporcionado minimamente a investigação com dois ou mais agentes, são estudos que não previam qualquer capacitação (ou programa ou intervenção), classificados na categoria (2). Nesse escopo, são propostas sugestões para que estudos futuros criem condições para o desenvolvimento de estratégias que promovam a cooperação e a interação entre os diversos agentes educacionais.

Evidentemente, por se tratar de um estudo de revisão bibliográfica sistemática, não foi pretensão deste trabalho esgotar a literatura nacional em relação à temática investigada. Além de que, foram propostos critérios para a busca dos estudos em periódicos com Qualis A1 e A2, nas áreas de Psicologia e Educação e, nesse contexto, algum estudo pode não ter sido recuperado, devido à publicação em algum outro periódico com Qualis diferente do proposto como critério. Sugere-se que sejam investigados estudos em outros periódicos, de modo a ampliar o sistema de busca. Do mesmo modo, outra limitação identificada refere-se ao processo de busca dos estudos, por intermédio do sistema por palavra-chave "inclusão escolar" e "inclusão educacional". É possível que outros estudos tenham sido publicados com outros termos. Todavia, a palavra-chave "inclusão escolar" parece estar mais associada à temática proposta.

Como o objetivo da busca referiu-se aos periódicos nacionais, é possível que algum estudo brasileiro tenha sido publicado em periódicos internacionais e, por essa razão, não foi contemplado na amostra investigada. Mesmo diante das limitações identificadas, ainda assim, o estudo alcançou o objetivo de mapear os estudos nacionais em relação ao envolvimento dos agentes no processo de inclusão escolar, tal qual de discutir as prováveis atuações desses distintos agentes envolvidos em tal processo e a importância do trabalho em conjunto entre eles, de modo a assegurar o processo de ensino e aprendizagem de todos os aprendizes. 
Referências
Almeida-Verdu, A. C. M., Fernandes, M. C., \& Rodrigues, O. M. P. R. (2002). A inclusão de pessoas com necessidades educativas especiais: implementação de práticas inclusivas e aspectos de planejamento educacional. Interação em Psicologia, 6(2), 223-231.

Aspilicueta, P., Leite, C. D., Rosa, E. C. M., \& Cruz, G. C. (2013). A questão linguística na inclusão escolar de alunos surdos: ambiente regular inclusivo versus ambiente exclusivamente ouvinte. Revista Brasileira de Educação Especial, 19(3), 395-410.

Bagaiolo, L., Guilhardi, C., \& Romano, C. (2005). Inclusão escolar sob a perspectiva da análise do comportamento. In H. Guilhardi, \& N. C. Aguirre (Eds.), Sobre comportamento e cognição: expondo a variabilidade (pp. 380392). Campinas, SP: ESETec.

Bardy, L. R., Hayashi, M. C. P. I., Schlunzen, E. T. M., \& Seabra Jr, M. O. (2013). Objetos de aqprendizagem como recurso pedagógico em contextos inclusivos: subsídios para a formação de professores a distância. Revista Brasileira de Educação Especial, 19(2), 273-288.

Benitez, P., \& Domeniconi, C. (2014). Capacitação de agentes educacionais: proposta de desenvolvimento de estratégias inclusivas. Revista Brasileira de Educação Especial. 20(3), 371-386.

Brasil. (2013). Ministério da Educação. Lei de diretrizes e bases da educação nacional (8a ed). Brasília, DF: MEC, 2013. Recuperado de http://bd.camara.gov.br/bd/ bitstream/handle/bdcamara/2762/ldb_8. ed.pdf? sequence $=1$

(1997). Ministério da Educação. Parâmetros curriculares nacionais $\left(1^{\mathrm{a}}\right.$ a $4^{\mathrm{a}}$ séries). Brasília, DF: MEC/SEF.

(2008). Ministério da Educação. Secretaria de Educação Especial. Política nacional de educação especial na perspectiva da educação inclusiva. Recuperado de http://portal.mec.gov.br/seesp/arquivos/pdf/ politica.pdf

Cappelini, V. L. M. F., \& Rodrigues, O. M. P. R. (2009). Concepções de professores acerca dos fatores que dificultam o processo da educação inclusiva. Educação, 32(3), 355-364.

Capellini, V. L. M. F., Rodrigues, O. M. P. R., Valle, T. G., Melchiori, L. B.; Zanata, E. M., Leite, L. P., \& Lepre, R. M. (2011). Perfil e avaliação dos participantes de um curso de formação continuada em práticas educacionais inclusivas na modalidade a distancia. Práxis Educativa, 6(1), 79-89.

Christovam, A. C. C., \& Cia, F. (2013). O envolvimento parental na visão de pais e professores de alunos com necessidades educacionais especiais. Revista Brasileira de Educação Especial, 19(4), 563-582.

Cruz, G. C., Aspilicueta, P., \& Leite, C. D. (2009). Escolarização de pessoas surdas na escola regular: explorando aspectos da comunicação na inclusão escolar. Práxis Educativa, 4(2), 169-176.

Cruz, G. C., Schneckenberg, M., Tassa, K. O. M., \& Chaves, L. (2011). Formação continuada de professores inseridos em contextos educacionais inclusivos. Educar em Revista, $42,229-243$.

Farias, I. M., Maranhão, R. V. A., \& Cunha, A. C. B. (2008). Interação professor-aluno com autismo no contexto da educação inclusiva: análise do padrão de mediação do professor com base na teoria da experiência da aprendizagem mediada. Revista Brasileira de Educação Especial, 14(3), 365-384.

França, E. S., \& Munford, D. (2012). Diferenças na sala de aula: conhecendo a prática pedagógica de duas professoras de ciências. Investigações em Ensino de Ciências, 17(3), 593-614.

Gena, A. (2006). The effects of prompting and social reinforcement on establishing social interactions with peers during the inclusion of four children with autism in preschool. International Journal of Psychology, 41(6), 541-554.

Gomes, C., \& Rey, F. L. G. (2007). Inclusão escolar: representações compartilhadas de profissionais da educação acerca da inclusão escolar. Psicologia: Ciência e Profissão, 27(3), 406-417.

Gomes, C., \& Souza, V. L. T. (2012). Psicologia e inclusão escolar: reflexões sobre o processo 
de subjetivação de professores. Psicologia: Ciência e Profissão, 32(2), 588-603.

Gomes, C. G. S., \& Mendes, E. G. (2010). Escolarização inclusiva de alunos com autismo na rede municipal de ensino de Belo Horizonte. Revista Brasileira de Educação Especial, 16(3), 375-396.

Guarinello, A. C., Berberian, A. P., Santana, A. P., Massi, G., \& Paula, M. (2006). A inserção do aluno surdo no ensino regular: visão de um grupo de professores do Estado do Paraná. Revista Brasil, 12(3), 317-330.

Gusmão, F. A. F., Martins, T. G., \& Luna, S. V. (2011). Inclusão escolar como uma prática cultural: uma análise baseada no conceito de metacontingência. Psicologia da Educação, $32,69-87$

Jesus, D. M., Barreto, M. A. S. C., \& Gonçalves, A. F. S. (2011). A formação do professor olhada no/pelo GT-15 - Educação Especial da Anped: desvelando pistas. Revista Brasileira de Educação Especial, 17(n esp 1), 77-92.

Lacerda, C. B. F. (2006). A inclusão escolar de alunos surdos: o que dizem alunos, professores e intérpretes sobre esta experiência. Caderno Cedes, 26(69), 163-184.

Leonardo, N. S. T., Bray, C. T., \& Rossato, S. P. M. (2009). Inclusão escolar: um estudo acertca da implantação da proposta em escolas de ensino básico. Revista Brasileira de Educação Especial, 15(2), 289-306.

Lima, S. F. B., Carreiro, L. R. R., Seraceni, M. F. F., Khoury, L. P., Braga, A. C., Araújo, M. V., \& Teixeria, M. C. T. V. (2012). Inattention and hyperactivity behavioral pattern of a child with Williams Syndrome: comparisons of regular and experimental class setting. Clinical Case Studies, 11(4) 312-325.

Luiz, F. M. R., Pfeifer, L. I., Sigolo, S. R. C. L., \& Nascimento, L. C. (2012). Inclusão de crianças com síndrome de Down. Psicologia em Estudo, 17(4), 649-658.

Mendes, E. G., Almeida, M. A., \& Toyoda, C. Y. (2011). Inclusão escolar pela via da colaboração entre educação especial e educação regular. Educar em Revista, 41(1), 81-93.
Monteiro, A. P. H., \& Manzini, E. J. (2008) Mudanças nas concepções do professor do ensino fundamental em relação à inclusão após a entrada de alunos com deficiência em sua classe. Revista Brasileira de Educação Especial, 14(1), 35-52.

Musis, C. R., \& Carvalho, S. P. (2010). Representações sociais de professores acerca do aluno com deficiência: a prática educacional e o ideal do ajuste à normalidade. Educação \& Sociedade, 31(110), 201-217.

Naiff, L. A. M., Ferreira, M. C., \& Naiff, D. G. M. (2013). Bem-estar profissional de professores de escolas públicas e privadas. Arquivos Brasileiros de Psicologia, 65(2), 288-303.

Oliveira, M. L., Antunes, A. M., Rocha, T. L., \& Teixeira, S. M. (2011). Educação inclusiva e a formação de professores de ciências: o papel das universidades federais na capacitação dos futuros educadores. Ensaio Pesquisa em Educação em Ciências, 13(3), 99-117.

Pelosi, M. B., \& Nunes, L. R. O. (2009). Caracterização dos professores itinerantes, suas ações na área de tecnologia assistiva e seu papel como agente de inclusão escolar. Revista Brasileira de Educação Especial, 15(1), 141-154.

Pereira, L. L. S., \& Benite, A. M. C. (2012). Redes sociais como espaço de interações discursivas sobre formação de professores de ciências para a educação inclusiva. Investigações em Ensino de Ciências, 17(3), 615-639.

Rodrigues, I. B., Moreira, L. E. V., \& Lerner, R. (2012). Análise institucional do discurso de professores de alunos diagnosticados como autistas em inclusão escolar. Psicologia: Teoria e Prática, 14(1), 70-83.

Rolfsen, A. B., \& Martinez, C. M. S. (2008). Programa de intervenção para pais de crianças com dificuldades de aprendizagem: um estudo preliminar. Paidéia, 18(39), 175-188.

Sampaio, C. S. (2006). A presença de uma aluna surda em uma turma de ouvintes possibilidade de (re)pensar a mesmidade e a diferença no cotidiano escolar. ETD Educação Temática Digital, 7(2), 47-57. 
Sanches, A. C. G., \& Oliveira, M. A. F. (2011). Educação inclusiva e alunos com transtorno mental: um desafio interdisciplinar. Psicologia: Teoria e Pesquisa, 27(4), 411-418.

Sant'Ana, I. M. (2005). Educação inclusiva: concepções de professores e diretores. Psicologia em Estudo, 10(2), 227-234.

Sekkel, M. C., Zanelatto, R., \& Brandão, S. B. (2010). Uma questão para a educação inclusiva: expor-se ou reguardar-se? Psicologia: Ciência e Profissão, 30(2), 296-307.

Silva, A. M., \& Mendes, E. G. (2012). Psicologia e inclusão escolar: novas possibilidades de intervir preventivamente sobre problemas comportamentais. Revista Brasileira de Educação Especial, 18(1), 53-70.

Silva, C. L., \& Leme, M. I. S. (2009). O papel do diretor escolar na implantação de uma cultura educacional inclusiva. Psicologia: Ciência e Profissão, 29(3), 494-511.

Silveira, F. F., \& Neves, M. M. B. J. (2006). Inclusão escolar de crianças com deficiência múltipla: concepção de pais e professores. Psicologia: Teoria e Pesquisa, 22(1), 79-88.
Sousa, C. S., \& Candeias, A. (2005). A colaboração intersectorial e multiprofissional na intervenção com crianças com necessidades especiais. Educação, 1(55), 11-30.

Toledo, E. H., \& Vitaliano, C. R. (2012). Formação de professores por meio de pesquisa colaborativa com vistas à inclusão de alunos com deficiência intelectual. Revista Brasileira de Educação Especial, 18(2), 319-336.

Unesco. (1994). Declaração de Salamanca. Recuperado de http://portal.mec.gov.br/ seesp/arquivos/pdf/salamanca.pdf

Unicef Brasil. (1990). Declaração mundial sobre educação para todos (conferência de Jomtien - 1990). Recuperado de http://www. unicef.org/brazil/pt/resources_10230.htm

Vitaliano, C. R. (2007). Análise da necessidade de preparação pedagógica de professores de cursos de licenciatura para inclusão de alunos com necessidades educacionais especiais. Revista Brasileira de Educação Especial, 13(3), 399-414.

Zanotto, M. L. B. (2000). Formação de professores: contribuição da análise do comportamento. São Paulo, SP: EDUC. 


\section{Priscila Benitez}

Doutoranda no Programa de Pós-Graduação em Psicologia da Universidade Federal de São Carlos, São Carlos - SP. Brasil.

Integrante do Instituto Nacional de Ciência e Tecnologia sobre Comportamento, Cognição e Ensino. Brasil.

E-mail: pribenitez@yahoo.com.br

\section{Camila Domeniconi}

Doutora em Educação Especial pela UFSCar. Docente adjunta da UFSCar, São Carlos - SP. Brasil.

Pesquisadora do Instituto Nacional de Ciência e Tecnologia sobre Comportamento, Cognição e Ensino. Brasil.

E-mail: camila@ufscar.br

\section{Endereço para envio de correspondência:}

Universidade Federal de São Carlos. Centro de Educação e Ciências Humanas, Departamento de Psicologia. Monjolinho. CEP: 13565-905. São Carlos - SP. Brasil.

O presente trabalho recebeu financiamento da bolsa de estudos de Doutorado, referente à primeira autora, concedido pela FAPESP (Processo $n^{\circ}$ 2010/16701-0).

Recebido: 12/05/2014, Aprovado: 30/09/2015. 